\title{
Is Cholecystectomy in Patients with Symptomatic Uncomplicated Cholelithiasis Beneficial in Improving the Lipid Profile?
} Adel Osman $^{1}$, Arwa H. Ibrahim
${ }^{1}$, Areej M. Alzamil ${ }^{1}$, Abdullah M. Alkhalifa ${ }^{1}$, Dania A. Badghaish ${ }^{2}$, Faisal
H. Al-dera ${ }^{3}$, Reda A. Alwosaibi ${ }^{1}$

1. Surgery, Imam Abdulrahman Bin Faisal University, Dammam, SAU 2. Internal Medicine, Imam Abdulrahman Bin Faisal University, Dammam, SAU 3. Surgery, King Fahad University Hospital, Dammam, SAU

Corresponding author: Adel Osman, ajosman@iau.edu.sa

\section{Abstract}

\section{Introduction}

Gallstone disease is an emerging health issue worldwide with its incidence on the rise. The development of gallstone disease is multifactorial, with risk factors including increased age, female sex, obesity, and the use of oral contraceptive pills. It has been established that more than $50 \%$ of patients with gallstone disease have a coexisting lipid disorder. Cholecystectomy, the definitive management of gallstones, may improve the lipid profiles of some patients.

\section{Objectives}

This study aims to examine the postoperative changes in the lipid profiles of patients who underwent cholecystectomy. These lipid profiles include levels of low-density lipoprotein (LDL), triglycerides (TG), high-density lipoprotein (HDL), total cholesterol (TC), and the Chol/HDL ratio.

\section{Methods}

This retrospective study included 55 patients who underwent cholecystectomy between 2013 and 2017. Biochemical parameters, which include LDL, TG, HDL, and TC levels, were collected using the hospital's recording system, in addition to the calculation of the Chol/HDL ratio.

\section{Results}

Statistically significant changes included a reduction in the mean LDL values in the two-, four-, and sixmonth postoperative periods ( $\mathrm{P}=0.029,0.000$, and 0.008 , respectively), increased mean TG levels one-week postoperatively $(\mathrm{P}=0.034)$, decreased mean TC levels at four $(\mathrm{P}=0.049)$ and six months $(\mathrm{P}=0.026)$ after cholecystectomy, and increased Chol/HDL ratio at two and 12 months postoperatively $(\mathrm{P}=0.03$, and 0.022 , respectively).

Received 01/13/2020

Review began 01/13/2020 Review ended 01/15/2020 Published 01/21/2020

\section{() Copyright 2020}

Osman et al. This is an open access article distributed under the terms of the Creative Commons Attribution License CC-BY 3.0., which permits unrestricted use, distribution, and reproduction in any medium, provided the original author and source are credited.

\section{Conclusions}

From the results, it can be concluded that cholelithiasis is associated with abnormal lipid profiles and that undergoing cholecystectomy may improve them and reduce the future risk of developing coronary artery disease. However, further research is needed to confirm this association.

Categories: General Surgery

Keywords: cholecystectomy, gallstone disease, lipid profile

\section{Introduction}

Gallstones disease is a chronic recurrent hepatobiliary disease. It is one of the emerging health problems worldwide and is becoming more common with an incidence of 1.4 per 100 persons [1]. Biliary stones are categorized into three main types: cholesterol, pigment, or mixed. Biliary stones formation is a complex process. It is governed by major factors: supersaturation of bile secreted, concentration of bile in the gallbladder, crystal nucleation, and abnormal gall bladder emptying [1]. Gallstone disease is an interaction between genetic and environmental factors. The prevalence increases with age; as an aging population, this justifies the rise in its rate. Females carry a higher incidence by two to three folds because of sex steroids and pregnancy changes. Oral contraceptives increase cholesterol secretion and decrease bile acids, resulting in the supersaturation of bile and increasing lithogenicity [2]. Presentation differs among patients, from asymptomatic and discovered incidentally. The most prevalent symptom is severe abdominal pain, requiring investigations and treatment. By the time they become symptomatic, many patients need surgical intervention. Based on the evidence, more than $50 \%$ of patients with gall stones have some sort of lipid disorder [1]. Though lipid and bile acids metabolisms are functionally correlated, how cholecystectomy 
affects lipid profile is not well-comprehended. High lipid profile readings, consisting of elevations in chylomicron, low-density lipoprotein (LDL), very-low-density lipoprotein (VLDL), and intermediate-density lipoprotein (IDL) levels, are becoming increasingly prevalent, especially with the spreading factors among the Saudi population, such as urban residence, increasing age, especially 40 years; physical inactivity, overweight and obesity, diabetes mellitus, frequent fast food consumption, and so on [3]. This, in turn, raises concerns about the effective management of such conditions. Cholecystectomy, which is considered the definitive management of symptomatic gallbladder diseases, for example, cholecystitis, biliary colic, gallbladder stones, and so on, was shown to decrease total serum lipids, serum cholesterol, serum LDL cholesterol, and serum triglycerides, and increase serum HDL cholesterol post-cholecystectomy after one day, one week, one month, and one year, according to Ali et al., Jindal et al., and Shen et al. [1,4-6]. Thus, the changes in plasma lipids are likely to have a significant effect on lowering the incidence of coronary artery disease development. Since the relationship between cholecystectomy and serum lipid profile has not been established yet, this study will investigate the serum lipid profiles of patients up to 12 months postcholecystectomy.

\section{Materials And Methods}

This retrospective study was carried out in the general surgery department at King Fahd Hospital of the University (KFHU) in Khobar, Saudi Arabia, from 2013 to 2017. The study was approved by the Institutional Review Board Committee of KFHU.

Fifty-five patients in the age group of 30-50 years, of both sexes, who underwent cholecystectomy were enrolled in this study. Patients with liver disease, nephrotic syndrome, diabetes mellitus, or familial dyslipidemias, those who didn't complete a follow-up of 12 months, and those who have a missing lipid profile were excluded from this study. Data were collected in regard to the lipid profile (LDL - TG - HDL - TC - Chol/HDL ratio) using the hospital's recording system. Preoperative (baseline), one week, two months, four months, six months, 8-months, 10 months, and 12 months are the intervals used for the lipid profile analysis.

Data analysis was performed using IBM SPSS Statistics for Windows, version 20 (IBM Corp., Armonk, NY). All categorical variables, including gender, nationality, and age groups, were expressed as frequencies and percentages. Mean and standard deviation were calculated for lipid profiles, including LDL, HDL, total cholesterol, triglycerides, and Chol/HDL. The paired sample student $\mathrm{t}$-test was used to compare the baseline mean values of the lipid profile with different follow-ups. P-value $<0.05$ was considered significant.

\section{Results \\ Demographic data}

As shown in Table 1, of the 55 included patients, there were 17 males (30.9\%) and 38 females (69.1\%). The mean age of the patients was $46.5 \pm 13.3$ years. Eight patients $(14.5 \%)$ aged 30 years and younger, 24 patients (43.6\%) aged from 31 to 50 years, and 23 patients (41.8\%) were older than 50 years. The nationalities included were Saudi (42 patients, $76.4 \%$ ), Egyptian (5 patients, 9.1\%), Filipino (3 patients, 5.5\%), Pakistani (2 patients, 3.6\%), and other (3 patients, 5.5\%). 


\section{Cureus}

\begin{tabular}{|c|c|c|}
\hline & Frequency & Percent \\
\hline \multicolumn{3}{|l|}{ Gender } \\
\hline Male & 17 & 30.9 \\
\hline Female & 38 & 69.1 \\
\hline \multicolumn{3}{|l|}{ Age (years) } \\
\hline$<30$ & 8 & 14.5 \\
\hline $31-50$ & 24 & 43.6 \\
\hline$>50$ & 23 & 41.8 \\
\hline \multicolumn{3}{|l|}{ Nationainty } \\
\hline Saudi & 42 & 76.4 \\
\hline Egyptian & 5 & 9.1 \\
\hline Filipino & 3 & 5.5 \\
\hline Pakistani & 2 & 3.6 \\
\hline Others & 3 & 5.5 \\
\hline
\end{tabular}

TABLE 1: Demographic characteristics of cases $(n=55)$

\section{Biochemistry}

Data were analyzed at baseline (preoperative) and the following postoperative intervals: one week, two months, four months, six months, eight months, 10 months, and 12 months, as shown in Table 2 and Table 3.

\begin{tabular}{|c|c|c|c|c|c|}
\hline & \multicolumn{5}{|l|}{$($ Min - Max) Mean \pm SD } \\
\hline & LDL & HDL & Total Cholesterol & Triglycerides & Chol/HDL \\
\hline Baseline & $120 \pm 38.2(36-193)$ & $45.8 \pm 11.9(26-77)$ & $180 \pm 51.6(34-271)$ & $128.1 \pm 66.5(41-382)$ & $4.33 \pm 1.42(2-8.3)$ \\
\hline After 1 week & $115.8 \pm 36.7(50-179)$ & $42.7 \pm 11.2(19-67)$ & $176.3 \pm 45.8(101-256)$ & $139.2 \pm 62.4(75-328)$ & $4.45 \pm 1.69(2.3-8.6)$ \\
\hline After 2 months & $101.2 \pm 39.4(45-178)$ & $46.2 \pm 15.1(28-80)$ & $178.5 \pm 52.8(98-246)$ & $123.6 \pm 41.7(23-186)$ & $3.92 \pm 1.22(2.4-6.9)$ \\
\hline After 4 months & $108.4 \pm 40.8(41-165)$ & $50 \pm 14.4(30-69)$ & $170.5 \pm 45.6(93-219)$ & $93 \pm 24.2(65-141)$ & $3.53 \pm 0.98(2.3-5.5)$ \\
\hline After 6 months & $108.5 \pm 41.3(43-172)$ & $46.2 \pm 13.6(29-76)$ & $173.2 \pm 51.1(100-268)$ & $115.5 \pm 62.1(45-263)$ & $3.96 \pm 1.34(2.3-6.5)$ \\
\hline After 8 months & $104.6 \pm 32.9(38-158)$ & $50.4 \pm 10(36-72)$ & $173 \pm 41.8(84-232)$ & $116.7 \pm 51.2(50-203)$ & $13.63 \pm 24.69(2.3-82)$ \\
\hline After 10 months & $93.7 \pm 33.1(53-158)$ & $47.5 \pm 14.3(28-73)$ & $159.9 \pm 39.7(100-229)$ & $114.4 \pm 46.1(55-177)$ & $3.53 \pm 0.89(2.3-4.8)$ \\
\hline After 12 months & $114.4 \pm 36.9(54-176)$ & $46 \pm 11.5(30-68)$ & $176.6 \pm 45(112-251)$ & $116.1 \pm 46.2(55-204)$ & $3.92 \pm 0.96(2.5-5.6)$ \\
\hline
\end{tabular}

TABLE 2: Lipid profile before and after surgery $(n=55)$

LDL: low-density lipoprotein; HDL: high-density lipoprotein 


\section{Cureus}

\begin{tabular}{|l|lllll|}
\hline \multicolumn{7}{|l|}{ P-Values* } & & & & \\
\hline & LDL & HDL & Total Cholesterol & Triglycerides & Chol/HDL \\
\hline Baseline Vs 1 week & 0.35 & 0.089 & 0.343 & 0.034 & 0.186 \\
\hline Baseline Vs 2 Months & 0.029 & 0.124 & 0.716 & 0.204 & 0.03 \\
\hline Baseline Vs 4 Months & 0.082 & 1 & 0.049 & 0.968 & 0.826 \\
Baseline Vs 6 Months & 0.008 & 0.54 & 0.026 & 0.844 & 0.191 \\
\hline Baseline Vs 8 Months & 0.18 & 0.429 & 0.568 & 0.551 & 0.241 \\
\hline Baseline Vs 10 Months & 0.077 & 0.563 & 0.82 & 0.75 & 0.104 \\
\hline Baseline Vs 12 Months & 0.328 & 0.849 & 0.955 & 0.168 & 0.022 \\
\hline
\end{tabular}

TABLE 3: Pairwise comparison of lipid profile before and after surgery $(n=55)$

Serum LDL Cholesterol

Statistical significance was found at the two, four, and six-month intervals $(P=0.029,0.000$, and 0.008 , respectively). The baseline serum LDL ranged from $36-193 \mathrm{mg} / \mathrm{dL}$, with a mean of $120 \pm 38.2 \mathrm{mg} / \mathrm{dL}$. The two months' range was $45-178 \mathrm{mg} / \mathrm{dL}$ with a mean of $101.2 \pm 39.4 \mathrm{mg} / \mathrm{dL}$. The four months' range was 41 $165 \mathrm{mg} / \mathrm{dL}$, with a mean of $108.4 \pm 40.8 \mathrm{mg} / \mathrm{dL}$, and the six months range was $43-172 \mathrm{mg} / \mathrm{dL}$, with a mean of $108.5 \pm 41.3 \mathrm{mg} / \mathrm{dL}$

Triglycerides

Triglycerides was the first element of the lipid profile to change, and it showed a statistically significant increase one week postoperatively, as its range was $75-328 \mathrm{mg} / \mathrm{dL}$, with a mean of $139.2 \pm 62.4 \mathrm{mg} / \mathrm{dL}$ (P $=0.034$ ) while the baseline ranged from $41-382 \mathrm{mg} / \mathrm{dL}$, with a mean of $128.1 \pm 66.5 \mathrm{mg} / \mathrm{dL}$.

\section{Serum HDL Cholesterol}

Although differences between the pre- and postoperative HDL were not statistically significant $(\mathrm{P}>0.05)$, HDL preoperatively ranged from $26-77 \mathrm{mg} / \mathrm{dL}$, with a mean of $45.8 \pm 11.9 \mathrm{mg} / \mathrm{dL}$, and deceased slightly one week postoperatively, range $19-67 \mathrm{mg} / \mathrm{dL}$, with a mean of $42.7 \pm 11.2 \mathrm{mg} / \mathrm{dL}(\mathrm{P}=0.089)$.

\section{Total Cholesterol}

The TC baseline ranged from $34-271 \mathrm{mg} / \mathrm{dL}$, with a mean of $180 \pm 51.6 \mathrm{mg} / \mathrm{dL}$. There were statistically significant decreases in the four and six-month periods postoperatively. The four months' range was 93 $219 \mathrm{mg} / \mathrm{dL}$, with a mean of $170.5 \pm 45.6(\mathrm{P}=0.049)$, whereas the six-months' range was $100-268 \mathrm{mg} / \mathrm{dL}$, with a mean of $173.2 \pm 51.1(\mathrm{P}=0.026)$.

Chol/HDL Ratio

The mean baseline of the Chol/HDL ratio was $4.33 \pm 1.42$ and ranged from $2-8.3$. It had a statistically significant increase in the two and 12 -month postoperative periods, as its mean value in the two-month interval was $3.92 \pm 1.22$, and it ranged from $2.4-6.9$. The mean $\mathrm{Chol} / \mathrm{HDL}$ ratio at 12 months ranged from 2.5 - 5.6, with a mean of $3.92 \pm 0.96(\mathrm{P}=0.03$ and 0.022 , respectively).

\section{Discussion}

Gallstone disease is a multifactorial disorder caused by the interaction between environmental and genetic factors. In the present study, the following risk factors have been discussed: gender, age, and lipid profile abnormality.

In this study, females were more affected than males with a 2:1 ratio, which is consistent with other studies and explains the consideration of female gender as a risk factor for gallstone disease development $[2,7]$.

Regarding age, the literature proved that increasing age is considered a risk factor for gallstone disease. This study showed that the majority of patients were aged from 31 to 50 years old. This is consistent with the 
results of Ahi in which the majority of patients were in the fourth decade, followed by the third and fifth decades [1].

LDL cholesterol levels decreased post-cholecystectomy and were found to be statistically significant at two, four, and six months postoperatively. In agreement with previous results, which found a decrease within three days to six months [1,4-10]. However, in the articles by Haq and Sabanathan et al., there was a significant increase $[3,11]$. This decrease is explained by the increased secretion rate of bile acid and phospholipid postoperatively and the upregulation of the LDL-Apolipoprotein B (ApoB) receptor resulting in LDL endocytosis from the blood into hepatocytes [12]. Within the hepatocytes, LDL binds to lysosomes that induce cholesterol delivery to the intracellular cholesterol pool for bile synthesis, leading to a steady LDL reduction post-cholecystectomy.

Triglyceride levels were found to be significantly increased at one week postoperatively, with a statistically insignificant decrease at the one-month interval and thereafter. Interestingly, Kuldip Singh Ahi had similar findings of an increase in TG after one week postoperatively, and a significant decrease was observed one month postoperatively [1]. The increase in TG 1-week post-cholecystectomy may have resulted from one of three mechanisms:

A. Increased TG use as a source of energy during the catabolic phase of trauma and limited carbohydrates amounts

B. Increased catecholamine release that occurs due to either surgical trauma or in response to types of general anesthesia, which activate hormone-sensitive triacylglycerol lipase (HSL) leading to lipolysis, thus increasing glycerol and TG [13]

C. Lipolytic rate acceleration in subcutaneous adipose tissue in response to a perioperative glucose infusion that overrides the anti-lipolytic effect of the glucose-induced hyperinsulinemia; this indicates a high association between abdominal surgery and lipolysis [13]

The postoperative changes of HDL were not statistically significant, although other studies found significant increases in HDL post-cholecystectomy [1,7,9].

The decrease in serum total cholesterol was found significant at four and six months following cholecystectomy. Similarly, some articles found a decrease in serum total cholesterol at intervals ranging from three days to one month [1-2,5,7,9-10]. Conversely, Sabanathan et al. found a significant increase in total cholesterol levels postoperatively [11]. This accounted for the fact that TC is the precursor for bile acids, whose secretion rate significantly increased along with phospholipids after cholecystectomy, causing improvement in the bile composition and significantly decreased the bile acids pool that may occur as a result of rapid cycling around the enterohepatic circulation (EHC)/hour [12]. Additionally, the upregulation of Apo B/E receptors that may increase the endocytosis of cholesterol and other types of fat into hepatocytes leads to the further formation of bile acids.

The Chol/HDL ratio was significantly higher at the one-week interval and conversely decreased at the oneyear interval. The results of Jindal et al. and Alkataan et al., however, indicated that the Chol/HDL ratio was significantly reduced at the one-week and one-month intervals $[5,7]$.

It is worth noting that some studies found that patients gained weight and had a higher body mass index (BMI) after cholecystectomy. This may have been caused by increased caloric, lipid, and carbohydrate intake, which consequently led to worsening lipid profiles $[4,14]$.

It is a well-known fact that disturbances in the lipid profile, specifically hypercholesterolemia, hypertriglyceridemia, and low HDL levels, are associated with an increased risk of coronary artery disease (CAD) and stroke, raising questions on whether an improvement in lipid profile following cholecystectomy may help in the future prevention of CAD and stroke. This was discussed by Alkataan et al., who calculated the atherogenic index pre- and post-cholecystectomy and found it to decrease significantly [7]. On the other hand, cholecystectomy has been found to be associated with increased cardiovascular risk factors. This could be explained by a strong association with metabolic syndrome [6,15].

\section{Conclusions}

Cholelithiasis is associated with an abnormal lipid profile that significantly improved after cholecystectomy. This improvement might continue with time; thus, it might be helpful in the prevention of subsequent CAD development. Therefore, the patient was advised to undergo cholecystectomy.

\section{Additional Information}

Disclosures 
Human subjects: Consent was obtained by all participants in this study. Imam Abdulrahman Bin Faisal University IRB issued approval IRB-2018-01-160. The application was reviewed and approved at the Imam Abdulrahman Bin Faisal University IRB meeting on Thursday, September 06, 2018. Animal subjects: All authors have confirmed that this study did not involve animal subjects or tissue. Conflicts of interest: In compliance with the ICMJE uniform disclosure form, all authors declare the following: Payment/services info: All authors have declared that no financial support was received from any organization for the submitted work. Financial relationships: All authors have declared that they have no financial relationships at present or within the previous three years with any organizations that might have an interest in the submitted work. Other relationships: All authors have declared that there are no other relationships or activities that could appear to have influenced the submitted work.

\section{Acknowledgements}

Our genuine thanks to the staff of the medical education department at King Fahad University Hospital, particularly Dr. Mona Al-Shaik, who helped in data statistics and analysis.

\section{References}

1. Ahi KS, Singh RP, Kaur H, Moudgil A: Serum lipid profile in pre and post cholecystectomy patients. Int J Anat Radiol Surg. 2017, 6:1-6.

2. Gill GS, Gupta K: Pre- and post-operative comparative analysis of serum lipid profile in patients with cholelithiasis. International journal of applied \& basic medical research. 2017, 7:186-188. 10.4103/2229516X.212968

3. Mujibul Haq AM, Giasuddin ASM, Jhuma KA, Choudhury MAM: Effect of cholecystectomy on lipid profile in Bangladeshi patients with cholelithiasis. J Metab Syndr. 2015, 5:192. 10.4172/2167-0943.1000192

4. Ali RB, Cahill RA, Watson RG: Weight gain after laparoscopic cholecystectomy. Ir J Med Sci. 2004, 173:9-12. 10.1007/bf02914515

5. Jindal N, Singh G, Ali I, Sali G, Reddy R: Effect of cholelithiasis and cholecystectomy on serum lipids and blood glucose parameters. Arch Int Surg. 2013, 3:97-101. 10.4103/2278-9596.122926

6. Shen C, Wu X, Xu C, Yu C, Chen P, Li Y: Association of cholecystectomy with metabolic syndrome in a Chinese population. PLoS One. 2014, 9:88189. 10.1371/journal.pone.0088189

7. Alkataan MAM, Bashi AYD, Al-Khyatt MK: Some serum lipid profile and glucose levels pre- and post cholecystectomy. J Bahrain Med Soc. 2010, 22:18-22.

8. Malik A, Wani ML, Iqbal Tak S, Irshad I, Ul-Hassan N: Association of dyslipidaemia with cholilithiasis and effect of cholecystectomy on the same. Int J Surg. 2011, 9:641-642. 10.1016/j.ijsu.2011.08.003

9. Moazeni-Bistgani M, Kheiri S, Ghorbanpour K: The effects of cholecystectomy on serum lipids during one year follow-up. Research. 2014, 1:1094. 10.13070/rs.en.1.1094

10. Kumar Y, Gola N, Kapoor V: Effect of cholecystectomy on serum lipid profile in gallstone patients: a comparative study. Int J Med Res Prof. 2018, 4:112-114.

11. Sabanathan S, Oomeer S, Jenkinson LR: Cholecystectomy or cholelithiasis - a missed marker for hyperlipidaemia? A combined retrospective and prospective study. Gastroent Res. 2008, 1:29-32. 10.4021/gr2008.11.1246

12. Zhao J-C, Xiao L-J, Zhu H, Shu Y, Cheng N-S: Changes of lipid metabolism in plasma, liver and bile during cholesterol gallstone formation in rabbit model. World J Gastroenterol. 1998, 4:337-339. 10.3748/wjg.v4.i4.337

13. Felländer G, Nordenström J, Tjäder I, Bolinder J, Arner P: Lipolysis during abdominal surgery. J Clin Endocrinol Metab. 1994, 78:150-155. 10.1210/jcem.78.1.8288698

14. Kenary AY, Yaghoobi Notash A Jr, Nazari M, et al.: Measuring the rate of weight gain and the influential role of diet in patients undergoing elective laparoscopic cholecystectomy: a 6-month follow-up study. Int J Food Sci Nutr. 2012, 63:645-648. 10.3109/09637486.2011.644767

15. Chavez-Tapia NC, Kinney-Novelo IM, Sifuentes-Renteria SE, et al.: Association between cholecystectomy for gallstone disease and risk factors for cardiovascular disease. Ann Hepatol. 2012, 11:85-89. 